\section{PWE-011 INDICATIONS AND OUTCOMES OF PATIENTS RECEIVING IN-PATIENT PARENTERAL NUTRITION: TYPE 2 IF PATIENTS ON HPN}

Lauren O'Flynn*, Nicky Wyer, Nicola Burch. University Hospitals Coventry and Warwickshire NHS Trust, Coventry, UK

\subsection{6/gutjnl-2019-BSGAbstracts.342}

Introduction Parenteral nutrition (PN) enables nutritional requirements to be met in cases of intestinal failure (IF) where enteral nutrition is insufficient or not possible. ${ }^{1}$ The main objective of our audit was to evaluate the indication, outcome, and survival of patients who received PN during their acute hospital admission.

Methods Data on in-patients receiving PN was collected prospectively using the Nutrition Team database. This was then retrospectively analysed, including review of overall outcome and mortality within 12-months of the in-patient spell. All patients receiving PN as an in-patient between January 2014 and December 2017 were included.

Patients were categorised according to type of IF: T1IF (inpatient $\mathrm{PN}<28$ days); T2IF (in-patient $\mathrm{PN}>28$ days; or inpatient $\mathrm{PN}<28$ days who were discharged on HPN with a plan to electively restore intestinal continuity later); and T3IF admissions (established HPN patients admitted acutely). Here we discuss the results of patients with T2IF requiring acute in-patient admission.

Results A total of 117 T2IF admissions were identified, in 98 patients; 44\% male; age 1-3 years (mean 55). In 25 admissions the patient received $\mathrm{PN}$ for $<28$ days; however all were discharged as new HPN patients (16 T2IF; 9 T3IF (6 palliative)). Duration of $\mathrm{PN}$ in the remaining patients was 2-53 days (mean 52). Cumulative duration of all T2IF admissions was 5219 days. Assuming average cost of an NHS bed day of $£ 222$ (NICE 20152); this equates to $£ 1,158,618$ (approx. $£ 289,655 / \mathrm{yr})$ in bed days without considering cost of inpatient treatment. 12 patients had multiple admissions with T2IF (-, mean 2); $58 \%$ of these occurred in the same year, $42 \%$ in separate years.

Indication for PN included: fistulae (21\%); obstruction (13\%); short bowel (9\%); failure of enteral nutrition $(8 \%)$; post-surgical complications (7\%); dysmotility (7\%); ischaemic bowel (5\%); malabsorption (5\%); pancreatitis (4\%); cancer (4\%); anastamotic leak (3\%); perforation (3\%); post-op ileus (3\%); gastric outlet obstruction (3\%); no access for enteral nutrition (3\%); crohn's (1\%); pre-op nutrition (1\%); planned IF surgery (1\%).

Outcome of T2IF was discharge on HPN in 55\%. Outcome in the remaining patients included: oral nutrition $(26 \%)$; NJ/Jejunostomy (9\%); NG (6\%); and RIP on PN (4\%). 12month follow-up data was available in 109 patients; 12-month survival was 77\%; overall survival to end August 2018 was $71 \%(\mathrm{n}=83)$.

Conclusions This audit demonstrates the significant financial cost and bed burden to centres managing patients with T2IF; and highlights the need for an IF tariff. In $45 \%$ patients there was return of intestinal function and resolution of intestinal failure, highlighting reversibility of T2IF. It was perhaps surprising that 12-month survival in this cohort was lower than the sub-analysis of all T3IF in-patient admissions $(77 \%$ versus $62 \%)$; this likely reflects that acute in-patient admission in patients with T3IF is a sign of disease progression/decompensation and therefore an indicator for reduced 12-month survival.

\section{REFERENCES}

1. Loris Pironi, et al.Espen guidelines on chronic intestinal failure in adult. Clin Nutr 2016;35:24-07;

2.. Costing statement: Implementing the NICE guideline on Transition between inpatient hospital settings and community or care home settings for adults with social care needs (NG27)

\section{PWE-012 INDICATIONS AND OUTCOMES OF PATIENTS RECEIVING IN-PATIENT PARENTERAL NUTRITION: TYPE 3 IF PATIENTS ON HPN}

Lauren O'Flynn*, Nicky Wyer, Nicola Burch. University Hospitals Coventry and Warwickshire NHS Trust, Coventry, UK

\subsection{6/gutjnl-2019-BSGAbstracts.343}

Introduction Parenteral nutrition (PN) enables nutritional requirements to be met in cases of intestinal failure (IF) where enteral nutrition is insufficient or not possible. ${ }^{1}$ The main objective of our audit was to evaluate the indication, outcome, and survival of patients who received PN during their acute hospital admission.

Methods Data on in-patients receiving PN was collected prospectively using the Nutrition Team database. This was then retrospectively analysed, including review of overall outcome and mortality within 12-months of the in-patient spell. All patients receiving $\mathrm{PN}$ as an in-patient between January 2014 and December 2017 were included.

Patients were categorised according to type of IF: T1IF (inpatient $\mathrm{PN}<28$ days); T2IF (in-patient PN>28 days; or inpatient $\mathrm{PN}<28$ days who were discharged on HPN with a plan to electively restore intestinal continuity later); and T3IF admissions (established HPN patients admitted acutely). Here we discuss the results of patients with T3IF requiring acute in-patient admission.

Results A total of 55 in-patient admissions were identified in 26 existing HPN patients (16 female; 10 male). Age ranged from 2-0 years (mean 60). Cause of IF (indication for HPN) was: short bowel syndrome (40\%); dysmotility (38\%); palliative cancer (13\%); mesenteric ischaemia (7\%); and malabsorption $(2 \%)$.

Duration of in-patient episode was -2 days (mean 16; cumulative total 875). Assuming average cost of an NHS bed day of $£ 222$ (NICE 20152); this equates to $£ 194,250$ in bed days without considering cost of in-patient treatment. Number of admissions per patient ranged from - (mean 2).

Indication for admission included sepsis (35\%); disease flare (22\%); elective surgery (13\%); elective admission to commence HPN (9\%); electrolyte derangement (7\%); cancer progression (4\%); GI Bleed (4\%); chemotherapy complications $(2 \%)$; fractured pelvis (2\%); overdose (2\%); and tube change $(1 \%)$. Source of sepsis included: urinary $(n=6)$, chest $(n=5)$, CRBSI $(n=3)$, discitis $(n=2)$, cholecystitis $(n=2)$, and abdominal collection $(n=1)$. Elective surgery included: venting PEG $(n=1)$; GI surgery e.g. intestinal continuity $(n=4)$; and non-GI surgery $(n=2)$.

Outcome of admission in the majority was discharge on HPN ( $n=49 ; 89 \%)$; one stopped HPN following continuity surgery. A total of 4 patients died during the admission (7\%), and 1 was commenced on the 'care of the dying' pathway (2\%). 12-month follow-up data was available in 54 of the admissions (98\%); 1 patient had only 9-months following last admission at the time of analysis. 12-month survival was $62 \%$ 
following admission (16/26); overall survival to end August 2018 was $54 \%(14 / 26)$.

Conclusions This audit highlights the significant cost and complexity of patients with T3IF on HPN requiring in-patient admission to hospital. With an increase in prevalence of T3IF of $20 \%$ per annum nationally, it is vital that HPN centres are sufficiently resourced and funded to facilitate management and care of this complex cohort of patients.

\section{REFERENCES}

1. Loris Pironi, et al.Espen guidelines on chronic intestinal failure in adult. Clin Nutr 2016;35:24-07;

2.. Costing statement: Implementing the NICE guideline on Transition between inpatient hospital settings and community or care home settings for adults with social care needs (NG27)

\section{PWE-013 NUTRITIONAL CARE PATHWAYS OF PATIENTS WITH MALIGNANT BOWEL OBSTRUCTION: EXPERIENCE FROM A UK TERTIARY-REFERRAL CENTRE}

${ }^{1}$ PS Patel", 'K Fragkos, ${ }^{1} \mathrm{~N}$ Keane, ${ }^{2} \mathrm{~K}$ Cauldwell, ${ }^{1} \mathrm{~F}$ O'Hanlon, 'J Rogers, 'S Obbard, ${ }^{1} \mathrm{~J}$ Barragry, ${ }^{1} \mathrm{G}$ Sebepos-Rogers, ${ }^{1} \mathrm{~S}$ Mehta, ${ }^{1} \mathrm{~F}$ Rahman, ${ }^{1} \mathrm{~S}$ Di Caro. ${ }^{1} \mathrm{G} /$ Services, University College London Hospital, London, UK; ${ }^{2}$ Palliative Care Central and North West London NHS Trust, London, UK

\subsection{6/gutjnl-2019-BSGAbstracts.344}

Introduction Emerging evidence is gathering for the use of parenteral nutrition $(\mathrm{PN})$ in patients with malignant bowel obstruction (MBO) who have lost nutritional autonomy and developed Intestinal Failure (IF). There is limited evidence describing the outcomes for $\mathrm{MBO}$ patients who are not referred for PN. We aimed to examine nutritional care pathways of $\mathrm{MBO}$ patients by referral for $\mathrm{PN}$ and appropriateness of referral/non-referral for PN.

Methods Retrospective cohort study of adults ( $\geq 18 \mathrm{yrs}$ ) admitted to University College London Hospital, admitted with MBO between 1.1.16- 31.12.16 with any readmissions up to 31.12.17. Data were analysed by comparing patients who were referred $(\mathrm{R})$ and not referred (NR) for PN.

Results 72 patients with $117 \mathrm{MBO}$ admissions (mean \pm SD age: $63.1 \pm 13.1 \mathrm{yrs}, 79 \%$ female), with median no. of admissions/ patient: 1 (range: 1-6). 24/72 patients were in R group. Predominant primary malignancies were gynaecological and gastrointestinal (76\%). 83\% of $\mathrm{MBO}$ patients had metastases and $61 \%$ were located subdiaphragmatically. All patients were at high risk of malnutrition using UCLH nutrition screening tool (score $=8$, a score of $\geq 7$ indicates high risk of malnutrition) and mean weight loss on admission was $7 \%$. Discussion of $\mathrm{PN}$ at $\mathrm{MDT}$ (21 vs. $4 \%, \mathrm{P}=0.02$ ) and dietetic contact (94 vs $41 \%, \mathrm{P}<0.0001)$ were more likely to occur in the $\mathrm{R}$ group. In 13/69 $\mathrm{MBO}$ admissions in NR group, the reasons for nonreferral to the Nutrition team are unclear. 20/24 referred patients received inpatient $\mathrm{PN}$, and 10 patients went home with PN. The remaining patients did not go home on PN as $\mathrm{BO}$ resolved or they were approaching the end of life. There were no differences in weight or BMI by PN referral groups. In all patients, median weight on admission was $55 \mathrm{~kg}$ (range: $3-00)$, and $5-5.8 \mathrm{~kg}$ at $0-3-$ and 3-6- months follow-up. Overall survival was 4.7 (1.-5.2) months, with no differences by referral groups (Fig 1).

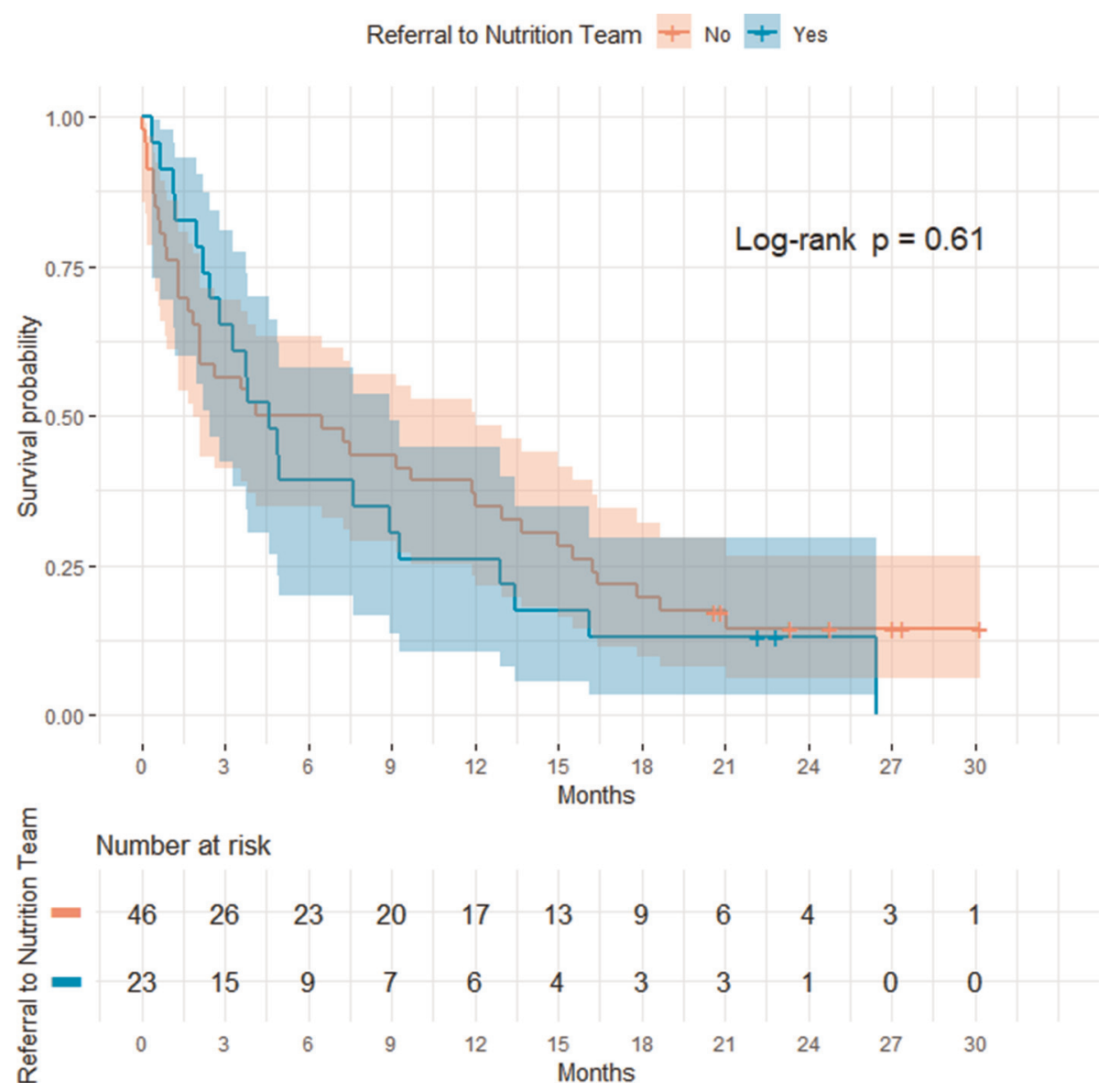

Abstract PWE-013 Figure 1 Kaplan Meier Curve of survival by referral for parenteral nutrition. 